\title{
Mounting an immune response correlates with decreased androgen levels in male peafowl, Pavo cristatus
}

\author{
Albert F. H. Ros · Maria Correia · John C. Wingfield • \\ Rui F. Oliveira
}

Received: 24 August 2007/Accepted: 30 May 2008

(C) Japan Ethological Society and Springer 2008

\begin{abstract}
Studies testing the "immunocompetence handicap hypothesis" have focussed on the immunosuppressive effects of androgens. Several recent studies have reported that mounting a humoral immune response might also result in a decrease in circulating androgen levels via a "negative feedback" on the hypothalamus-pituitarygonadal axis (HPG). The aim of this correlative study was to analyse these immunosuppressive and HPG-suppressive interactions in reproductively active males of the peafowl. We collected blood samples of free living birds before and after challenging the immune system with a non-pathogenic antigen (sheep erythrocytes), and analysed immune parameters and plasma levels of the two main androgens in birds, testosterone and dihydrotestosterone. Males displaying larger versions of the main secondary sexual trait, the long and conspicuously ornamented train, tended to have higher androgen levels and significantly lower circulating levels of leukocytes, indicating that exaggerated ornaments might signal properties of the endocrine and immune system. Actual circulating levels of androgens did not correlate with the plasma levels of leukocytes and the antibody response to SRBC. However, changes in plasma levels of both androgens showed negative correlation with both leukocytes $(P<0.1)$ and SRBC responses $(P<0.05)$. The data therefore support the prediction that activity of the immune system is HPG-suppressive. Such suppression
\end{abstract}

A. F. H. Ros $(\bowtie) \cdot$ M. Correia · R. F. Oliveira

Unidade de Investigação em Eco-Etologia,

Instituto Superior de Psicologia Aplicada,

Rua Jardim do Tabaco 34, 1149-041 Lisbon, Portugal

e-mail: aros@ispa.pt

J. C. Wingfield

Department of Biology, University of Washington,

P.O. Box 351800, Seattle, WA 98195-1800, USA has been proposed to be especially costly during the reproductive season, during which androgens facilitate the expression of exaggerated traits that play an important role in sexual competition.

Keywords Peacock - Sexual traits - Immunocompetence · Testosterone $\cdot$ Dihydrotestosterone $\cdot$ SRBC .

Heterophil-lymphocyte ratio

\section{Introduction}

Males often express multiple sexual ornaments and displays, some of which correlate with the quality of the holder to cope with or to resist parasite infection (Hamilton and Zuk 1982; Getty 2002). Such traits are likely to have evolved through sexual selection and have associated viability costs (see Zahavi 1975; Andersson 1994). In the "immunocompetence handicap hypothesis", Folstad and Karter (1992) provided a causal mechanism for these viability costs by arguing that androgens act as a "doubleedged sword", stimulating the development of costly characteristics used in sexual selection, while debilitating viability by suppressing the immune system. This androgen-regulated immunocompetence handicap would ensure that only males in good health condition or having constitutive mechanisms ("genetic" resistance) to cope with virulent parasites would carry the sexual character.

Many studies testing the immunocompetence handicap have focussed on the primary prediction derived from the immunocompetence handicap hypothesis, namely that androgens are immunosuppressive and increase the prevalence of parasitic infections. A meta-analysis of these studies showed that the evidence for androgen-induced immunosuppression is ambiguous for birds (Roberts et al. 
2004). Fewer studies have taken into account that infections might have a suppressive effect on the expression of social and reproductive behavior via an effect of the immune system on the hypothalamus pituitary axis (see Hart 1990; Hillgarth and Wingfield 1997; Fox and Hudson 2001). That such an alternative pathway exists is suggested by a study in which male house finches, Carpodacus mexicanus, infected with coccidian parasites were found to have lower levels of testosterone than uninfected males (Duckworth et al. 2001). Furthermore, two recent studies have shown that experimental injections with sheep red blood cells (SRBC - a nonpathogenic antigen that stimulates a humoral antibody response) result in a decrease in testosterone levels (Garamszegi et al. 2004; Peters et al. 2004). Finally, treatment with cytokines has been shown to lower androgen production of the testis in goldfish (Lister and Van Der Kraak 2002). This alternative pathway was anticipated by Folstad and Karter (1992) in the immunocompetence handicap hypothesis as a "negative feedback loop" between the immune system and the endocrine system. It would be especially costly for males of polygynous species, in which androgen levels are constantly at physiological maximum in order to enhance their success in competitions for mating opportunities (Balthazart et al. 1983; Wingfield et al. 1990; Hirschenhauser and Oliveira 2006). A decrease in androgen production due to activity of the immune system could decline the competitive success of these reproductively active males.

The aim of this study was to analyse immune-androgen relationships in males of the peafowl (Pavo cristatus). We choose to study these relationships in the peafowl because this species has a polygynous mating system in which high male mating success is correlated with the exaggerated display of their long trains, which are conspicuously decorated with many colourful eye-spots (Petrie et al. 1991; Yasmin and Yahya 1996; Loyau et al. 2005b). Møller and Petrie (2002) showed that train length correlates negatively with antibody responses to SRBC and the relative number of lymphocytes in the blood (heterophil-lymphocyte ratio). In contrast, train length was positively correlated with cellular immunity, suggesting that males redistributed immune resources from humoral to peripheral cellular defence mechanisms (see Braude et al. 1999).

An experiment was carried out in which the humoral immune systems of peafowl males were challenged with SRBC antigen and the resulting changes in specific antibodies and leukocytes were measured. Further, we measured the plasma levels of the two androgens testosterone and dihydrotestosterone, which have a facilitating effect on male reproductive behavioural repertoire (Balthazart et al. 1983; Ball and Balthazart 2004). Both have high affinities to androgen receptors, though their effects can differ depending on the target tissue, since testosterone can be aromatized to estradiol or reduced to dihydrotestosterone, while dihydrotestosterone is not aromatizable. We anticipated two different scenarios derived from the immunocompetence handicap hypothesis which are not mutually exclusive: (1) androgens are immunosuppressive-actual androgen levels are negatively correlated with immune parameters; (2) activity of the immune system suppresses the HPG axis-antibody responses to the SRBC challenge are negatively correlated with changes in androgen levels.

\section{Methods}

Study design and animal handling

Between May and July 2001, Indian peafowl were studied in two parks in the city area of Lisbon, Portugal. The "Jardim da Estrela" park in Lisbon held a population of 12 free-roaming birds, and the "Jardim Marechal Carmona" park in Cascais held a population of 20 free-roaming birds. Both parks receive many visitors and therefore peafowl were highly accustomed to the presence of humans. To study the relationship between testosterone and immunocompetence, 14 peafowl males were caught twice (body weight $12.9 \pm 0.2 \mathrm{~kg}$ ). The birds were very tame, which allowed us to approach them closely and grab them by the legs and wings. At first capture, blood samples (within 5 min, $1.5 \mathrm{ml}$ ) were drawn from the brachial wing vein with a heparin-rinsed needle and syringe. Birds were weighed $( \pm 0.1 \mathrm{~kg})$ and colour-banded for individual recognition, and train length was measured to the nearest centimeter. Thereafter the birds were immunized (see below) and released. Birds were re-caught one week later in order to draw post-immunization blood samples.

\section{Haematological analysis}

One millilitre of the blood sample was sent to the "Laboratório Nacional de Investigação Veterinária" in Lisbon for haematological analyses. Hematocrite percentages were calculated using the "Wintrobe" method. A Neubauer counting chamber was used to count the number of leukocytes. The relative frequency of each family class of leukocytes was counted from blood smears. This percentage was multiplied by the total count of leukocytes to estimate the total count of each family of white blood cells. Total counts are expressed as number of cells per $\mathrm{mm}^{3}$ blood.

Immunization and haemagglutination assay

The measurement of antibody titre following immunization with sheep erythrocytes (SRBC) integrates a large number 
of immunological events ( $\mathrm{T}$ - and B-cell responses), including initial antigen recognition and presentation and the final production of specific antibodies (both $\operatorname{IgM}$ and IgG). Blood cells from one donor sheep were collected and used as SRBC antigens. The cells were washed three times in phosphate-buffered saline (PBS) and re-suspended in PBS at $5 \times 10^{8}$ cells $/ \mathrm{ml}(2 \% \mathrm{SRBC})$. Each bird was immunized with the antigen suspension by intraperitoneal injection. The amount of SRBC suspension that was injected was adjusted to the body mass of the bird $(0.5 \mathrm{ml}$ SRBC suspension/kg body mass, see Aitken and Parry 1974). In birds, primary antibody responses to SRBC immunization show peak values about one week after immunisation (Aitken and Parry 1974; Apanius 1998; Fair et al. 1999; Snoeijs et al. 2007). We therefore recaptured all birds one week later in order to draw the post-immunization blood samples.

To estimate antibody levels, the following procedure was used. Plasma contains complement proteins which, together with the antibody, induce the lyses of antigencarrying cells. To prevent complement from interfering with the haemagglutination reaction by inducing lyses of SRBC, the plasma was heated to $56{ }^{\circ} \mathrm{C}$ for $30 \mathrm{~min}$ in order to denaturize the complement. Thereafter, the plasma was diluted 1:1 in PBS and then serially diluted in PBS in U-shaped microtitre plates. An equal volume of $2 \%$ SRBC was added to these dilutions, and the plates were incubated at $37^{\circ} \mathrm{C}$ for $60 \mathrm{~min}$. Titres were scored visually as the highest twofold dilution of plasma showing haemagglutination and represented as integers on a $\log 2$ scale. All birds showed low pre-immunization antibody titres to SRBC (mean: $2.2 \pm 0.1$ ), which may be due to some cross-reactivity with low specific antibody (see Seto and Henderson 1968). The antibody response to SRBC treatment was calculated as a measure of immunocompetence as the postminus the pre-immunization score.

\section{Radioimmunoassays}

Blood was centrifuged and stored at $-30{ }^{\circ} \mathrm{C}$ before transport to the University of Washington. Plasma levels of the two most abundantly circulating androgens in birds, dihydrotestosterone and testosterone, were measured by radioimmunoassay after partial purification. Briefly, the plasma was equilibrated for at least $2 \mathrm{~h}$ with approximately $2,000 \mathrm{cpm}$ of tritiated dihydrotestosterone and testosterone (to determine the percent recovery after extraction and chromatography), and made up to $400 \mu \mathrm{l}$ with distilled water. Samples were extracted with $5 \mathrm{ml}$ of freshly redistilled dichloromethane, and the organic phase was aspirated and evaporated to dryness under a stream of nitrogen at $45^{\circ} \mathrm{C}$. Dried extracts were then transferred to diatomaceous earth columns in $0.5 \mathrm{ml}$ of $2 \%$ ethyl acetate in isooctane. Steroid fractions were then eluted with increasing concentrations of ethyl acetate in isooctane, as described by Wingfield and Farner (1975). Dihydrotestosterone and testosterone fractions were dried under a stream of nitrogen and measured by direct radioimmunoassay as described by Wingfield et al. (1991). Recoveries and intraassay variations were within the limits described by Wingfield and Farner (1975). All samples were processed in one assay, so inter-assay variation was not an issue.

Statistical analysis

In order to test correlations without making assumptions about the distribution of the data, Spearman rank correlation coefficients were calculated using the SPSS 13.0 package (SPSS Inc., Chicago, IL, USA). Some values were missing from the data because: (1) we were not able to capture two of the 14 animals for the second time; (2) some of the blood samples coagulated during transportation from the field to the laboratory; (3) some of the plasma samples were too small to perform all assays. To increase the statistical power we averaged leukocyte and androgen values over the two periods. $P$ values represent two-tailed probabilities, except for some comparisons in which we checked for relationships that were reported significant by Møller and Petrie (2002).

\section{Results}

As expected from earlier studies on peafowl, males with longer trains had less circulating leukocytes (Table 1 and Fig. 1). They also had higher heterophil-lymphocyte ratio (Table 1, H/L: $P=0.047$ ), indicating relatively low counts of lymphocytes in males with longer trains. Specific antibody titres to SRBC were significantly elevated after immunization $\left(2^{3.90}\right.$-fold increase, Table 1 ; Wilcoxon matched pair test: $W=3, n=11, P=0.005)$. Although the antibody response to $\mathrm{SRBC}$ showed the expected negative correlation with train length (Fig. 1) and the heterophillymphocyte ratio, the regression coefficients were far from statistically significant in this sample (Table $1, P=0.21$ and 0.26 , respectively). From the graphical representation (Fig. 1), it seems that males with longer trails expressed more variation in humoral immunity.

Both the average plasma levels of dihydrotestosterone and testosterone and the change in the individual patterns of dihydrotestosterone and testosterone during the experiment $(\delta \mathrm{DHT}$ and $\delta \mathrm{T})$ were significantly positively correlated ( $P<0.001$ and 0.038 , respectively). Heterophillymphocyte ratio was positively correlated with testosterone levels but not with dihydrotestosterone levels (Table 1, $P=0.05$ and 0.23 , respectively). Actual levels of both 
Table 1 Results from bivariate correlation analyses performed between immunocompetence and androgen levels

\begin{tabular}{|c|c|c|c|c|c|}
\hline & Mean \pm SEM & Train length & Leukocytes & $\mathrm{H} / \mathrm{L}$ ratio & SRBC \\
\hline Train length $(\mathrm{cm})$ & $154 \pm 4^{\mathrm{c}}$ & & & & \\
\hline Leukocyte count $\left(10^{3}\right.$ cells $\left./ \mathrm{mm}^{3}\right)$ & $17.8 \pm 0.9$ & $-0.78 * * *$ & & & \\
\hline Heterophil-lymphocyte ratio & $1.44 \pm 0.14$ & $0.48 * * 1$-sided & -0.11 & & \\
\hline SRBC antibody response & $3.90 \pm 0.80$ & $-0.22^{\mathrm{b}}$ & $0.22^{\mathrm{a}}$ & $-0.28^{\mathrm{a}}$ & \\
\hline DHT (ng/ml) & $0.82 \pm 0.17^{\mathrm{c}}$ & $0.20^{\mathrm{c}}$ & -0.10 & 0.36 & $-0.29^{\mathrm{b}}$ \\
\hline $\mathrm{T}(\mathrm{ng} / \mathrm{ml})$ & $2.14 \pm 0.23^{\mathrm{c}}$ & $0.47 * \mathrm{c}$ & -0.24 & $0.55 * *$ & $-0.19^{\mathrm{b}}$ \\
\hline$\delta$ DHT $(\mathrm{ng} / \mathrm{ml})$ & $0.39 \pm 0.46^{\mathrm{b}}$ & $0.64 * * \mathrm{c}$ & $-0.57 *^{\mathrm{a}}$ & $0.59^{* \mathrm{a}}$ & $-0.70 * * \mathrm{a}$ \\
\hline$\delta \mathrm{T}(\mathrm{ng} / \mathrm{ml})$ & $-0.57 \pm 0.42^{\mathrm{b}}$ & $0.52 * \mathrm{c}$ & $-0.58 *^{\mathrm{a}}$ & $0.41^{\mathrm{a}}$ & $-0.80 * * * \mathrm{a}$ \\
\hline
\end{tabular}

Leukocyte values are expressed as the cell count per $\mathrm{mm}^{3}$ of blood. $R$ values refer to Spearman rank correlation coefficients. $N=13$ or is annotated: ${ }^{\mathrm{a}} N=10,{ }^{\mathrm{b}} N=11,{ }^{\mathrm{c}} N=14$

$* P<0.10, * * P<0.05$, *** $P<0.01$

Fig. 1 Graphical representation of the correlation between train length $(\mathrm{m})$, antibody response to SRBC (change in haemagglutination titres on a $\log _{2}$ scale), leukocyte concentration (cells per $\mathrm{mm}^{3}$ ), and the change in the total plasma levels of the androgens (dihydrotestosterone + testosterone $\mathrm{ng} / \mathrm{ml}$ )
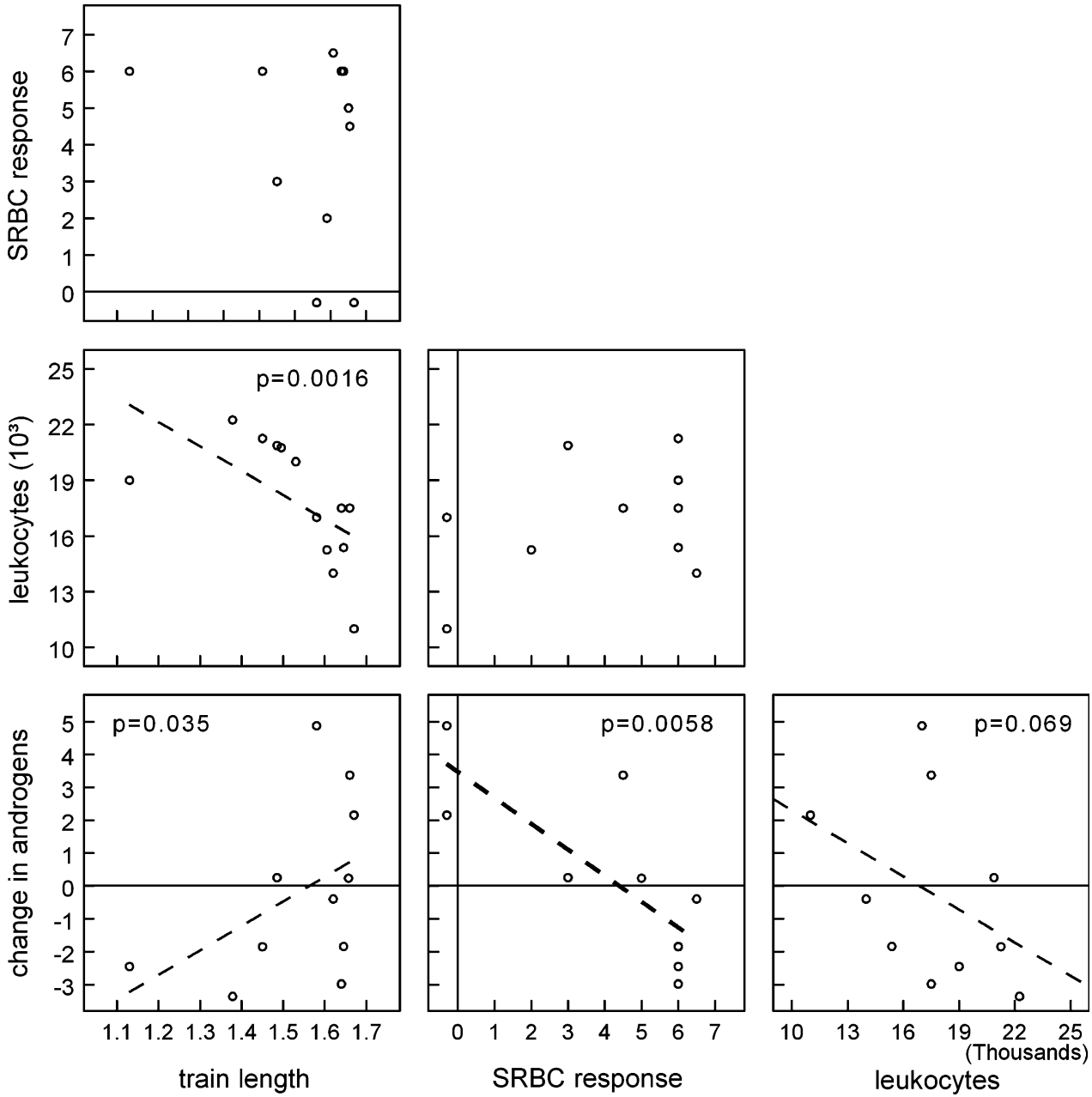

androgens were not correlated with the antibody response to SRBC (Table 1), which is not consistent with our first prediction that androgens are immunosuppressive. However, changes in both dihydrotestosterone and testosterone levels correlated negatively with the antibody response to SRBC immunization (Table 1: $P=0.023$ and 0.005 , respectively; see Fig. 1 for total androgen levels), which is consistent with our second prediction that the activity of the immune system is "HPG-suppressive". Furthermore, train length was positively correlated with changes in androgens $(P=0.026$ and 0.10 , respectively; see Fig. 1 for total androgen levels), and the graphical representation of 
this regression indicates that the variation in androgen levels was only suppressed in males with smaller train length, and thus in males that are likely to show poor mating success.

\section{Discussion}

Variations in the plasma levels of testosterone and the nonaromatizable androgen dihydrotestosterone were highly correlated, and correlative trends with secondary sexual characters and immunocompetence were in similar directions. Changes in dihydrotestosterone and testosterone, but not the actual levels of these androgens, were negatively correlated with the variation in specific antibody production resulting from SRBC immunization. This negative correlation is consistent with the results of two recent studies that used SRBC immunization to challenge the immune system. In the first study in male mallards (Anas platyrhynchos), Peters et al. (2004) found a negative correlation between changes in testosterone levels and antibody responsiveness. In the second study, Garamszegi et al. (2004) found that collared flycatchers (Ficedula albicollis) immunized with SRBC showed a decrease in testosterone levels and song rate in comparison with those that received placebo treatment. Together, these studies suggest that androgen levels vary as a function of the activity of the immune system.

Most studies testing the immunocompetence handicap hypothesis of Folstad and Karter (1992) have focussed on the immunosuppressive effects of androgens as a mechanism for the trade-off between secondary sexual characters and parasite resistance (for a review see Roberts et al. 2004). However, Folstad and Karter (1992) also anticipated a "negative feedback loop" of the immune system to the hypothalamus-pituitary-gonadal axis. They argued that its function is "to enable the individual to regulate testosterone profiles to changing internal demands". Such demands may be the energetic stress due to the production and proliferation of leukocytes in the immune organs resulting from an immune response being mounted to an infection (see Sheldon and Verhulst 1996; Svensson et al. 1998; Ots et al. 2001; Martin et al. 2003). In an action not necessarily unrelated to energy redistribution, immune cells involved in the inflammatory process may target endocrine glands and the brain through the release of interleukins (e.g. Weyts et al. 1999; Lister and Van Der Kraak 2002). When circulating levels of testosterone and dihydrotestosterone decrease as a result of such immune activity, this is likely to alter behavioural activity and this may have important negative consequences for reproductive success (Hart 1990; Poulin 1995; Weatherhead et al. 1995). In concordance with this interpretation, the injection of peafowl males with lipopolysaccharides of $E$. coli, which simulates a bacterial infection resulting in a general activation of the immune system, has been shown to cause a decrease in display rate (Loyau et al. 2005a).

Møller and Petrie (2002) published a detailed study of the correlations between different male sexual ornaments and immunocompetence. One of their results was that the size and complexity of the train negatively correlated with humoral and positively with cellular immunity. Moreover, in both the present study and their study, a positive correlation was found between trail length and heterophillymphocyte ratio. Thus males with long trails, a trait that correlates with high mating success (Petrie et al. 1991; Yasmin and Yahya 1996), have relatively low levels of lymphocytes. Lymphocytes are the cells that produce the specific antibodies against pathogens. The trends found in male peafowl may therefore reflect a redistribution of immune cells over two major arms of the immune system (see Braude et al. 1999): (1) the primary response against pathogens entering the body, which is governed by rapid cellular mechanisms at the periphery of the body, and (2) the humoral immune response, which provides a slow but more effective protection against the proliferation of pathogens in the body. Animals in good health may be able to afford to lower their humoral immunity when protected by cellular and constitutive mechanisms. This would make them less prone to humoral immunity-related suppression of androgen release, which would be compatible with their polygynous mating system (Wingfield et al. 1990).

In summary, the correlative data on the relation between immunity and androgen levels support the prediction that activity of the immune system suppresses androgen production. Reproductively active males may modulate their immune defences in order to minimize such androgensuppressive effects. Because it is likely that they may only do so when in good health, this pathway could therefore be a potent alternative immunocompetence handicap mechanism that results in females mating with high-quality males.

Acknowledgments We thank the administration of the "Jardim da Estrela" in Lisbon and the "Parque Marechal Carmona" in Cascais for kindly helping us during the study. We are grateful to Lynn Erckmann for expert help with the hormone assays, and to Dr. Maria da Luz Ferreira and Dr. Eugénia Rebelo of LNIV of the Ministry of Agriculture, Portugal, for carrying out the immunological assays. We further thank the reviewers and Dr. Yoshitaka Tsubaki for their constructive comments on earlier versions of the manuscript. During this study AFHR was supported by a postdoctoral fellowship from the Portuguese Foundation for Science and Technology (FCT, SFRH/ BPD/7143/2001). The research was further supported by FCT's Plurianual Program to AFHR (R\&D Unit 331/94), and a grant (IBN-9905679) from the National Science Foundation to JCW. The procedures used in this study comply with the Principles of Animal Care, publication No. 86-23, revised 1985, from the National Institutes of Health, USA, and with the current laws of Portugal. 


\section{References}

Aitken ID, Parry SH (1974) The comparative serological response of the chicken, pheasant and quail to soluble and a particulate antigen. Immunology 27:623-629

Andersson M (1994) Sexual selection. Princeton University Press, Princeton, NJ

Apanius V (1998) Ontogeny of immunity in birds. In: Starck JM, Ricklefs RE (eds) Avian growth and development: evolution within the altricial-precocial spectrum. Oxford University Press, New York, pp 203-222

Ball GF, Balthazart J (2004) Hormonal regulation of brain circuits mediating male sexual behavior in birds. Physiol Behav 83:329346

Balthazart J, Schumacher M, Ottinger MA (1983) Sexual differences in the Japanese quail: behavior, morphology and intracellular metabolism of testosterone. Gen Comp Endocrinol 51:191-207

Braude S, Tang-Martinez Z, Taylor GT (1999) Stress, testosterone, and the immunoredistribution hypothesis. Behav Ecol 10:345350

Duckworth RA, Mendonca MT, Hill GE (2001) A condition dependent link between testosterone and disease resistance in the house finch. Proc R Soc Lond B 268:2467-2472

Fair JM, Hansen ES, Ricklefs RE (1999) Growth, developmental stability and immune responses in juvenile Japanese quails (Coturnix coturnix japonica). Proc R Soc Lond B 266:17351742

Folstad I, Karter A (1992) Parasites, bright males, and the immunocompetence handicap. Am Nat 139:603-622

Fox A, Hudson PJ (2001) Parasites reduce territorial behaviour in red grouse (Lagopus lagopus scoticus). Ecol Lett 4:139-143

Garamszegi LZ, Møller AP, Torok J, Michl G, Peczely P, Richard M (2004) Immune challenge mediates vocal communication in a passerine bird: an experiment. Behav Ecol 15:148-157

Getty T (2002) Signaling health versus parasites. Am Nat 159:363371

Hamilton WD, Zuk M (1982) Heritable true fitness and bright birds: a role for parasites? Science 218:384-387

Hart BL (1990) Behavioral adaptations to pathogens and parasites: five strategies. Neurosci Biobehav Rev 14:273-294

Hillgarth N, Wingfield JC (1997) Testosterone and immunosupression in vertebrates: implications for parasite-mediated sexual selection. In: Beckage NE (ed) Parasites and pathogens: effects on host hormones and behavior. Chapman and Hall, New York, pp 143-155

Hirschenhauser K, Oliveira RF (2006) Social modulation of androgens in male vertebrates: meta-analyses of the challenge hypothesis. Anim Behav 71:265-277

Lister A, Van Der Kraak G (2002) Modulation of goldfish testicular testosterone production in vitro by tumor necrosis factor, interleukin-1, and macrophage conditioned media. J Exp Zool 292:477-486

Loyau A, Saint Jalme M, Cagniant C, Sorci G (2005a) Multiple sexual advertisements honestly reflect health status in peacocks (Pavo cristatus). Behav Ecol Sociobiol 58:552-557
Loyau A, Saint Jalme M, Sorci G (2005b) Intra- and intersexual selection for multiple traits in the peacock (Pavo cristatus). Ethology 111:810-820

Martin LBII, Scheuerlein A, Wikelski M (2003) Immune activity elevates energy expenditure of house sparrows: a link between direct and indirect costs? Proc R Soc Lond B 270:153-158

Møller AP, Petrie M (2002) Condition dependence, multiple sexual signals, and immunocompetence in peacocks. Behav Ecol 13:248-253

Ots I, Kerimov AB, Ivankina EV, Ilyina TA, Horak P (2001) Immune challenge affects basal metabolic activity in wintering great tits. Proc R Soc Lond B 268:1175-1181

Peters A, Delhey K, Denk AG, Kempenaers B (2004) Trade-offs between immune investment and sexual signaling in male mallards. Am Nat 164:51-59

Petrie M, Halliday T, Sanders C (1991) Peahens prefer peacocks with elaborate trains. Anim Behav 41:323-331

Poulin R (1995) "Adaptive" changes in the behaviour of parasitized animals: a critical review. Int J Parasitol 25:1371-1383

Roberts ML, Buchanan KL, Evans MR (2004) Testing the immunocompetence handicap hypothesis: a review of the evidence. Anim Behav 68:227-239

Seto F, Henderson WG (1968) Natural and immune hemagglutinin forming capacity of immature chickens. J Exp Zool 16:501-512

Sheldon BC, Verhulst S (1996) Ecological immunity: costly parasite defences and trade-offs in evolutionary ecology. TREE 11:317321

Snoeijs T, Eens M, Van-Den-Steen E, Pinxten R (2007) Kinetics of primary antibody responses to sheep red blood cells in birds: a literature review and new data from great tits and European starlings. Anim Biol 57:79-95

Svensson E, Råberg L, Koch C, Hasselquist D (1998) Energetic stress, immunosuppression and the costs of an antibody response. Funct Ecol 12:912-919

Weatherhead PJ, Metz KJ, Shutler D, Muma KE, Bennet GF (1995) Blood parasites and dominance in captive blackbirds. J Avian Biol 26:121-123

Weyts FAA, Cohen N, Flik G, Verburg-Van-Kemenade BML (1999) Interactions between the immune system and the hypothalamopituitary-interrenal axis in fish. Fish Shellfish Immunol 9:1-20

Wingfield JC, Farner DS (1975) The determination of five steroids in avian plasma by radioimmunoassay and competitive protein binding. Steroids 26:311-327

Wingfield JC, Hegner RE, Lewis D (1991) Circulating levels of luteinizing hormone and steroid hormones in relation to social status in the cooperatively breeding white-browed sparrow weaver, Plocepasser mahali. J Zool Lond 225:43-58

Wingfield JC, Hegner RE, Dufty AM Jr, Ball GF (1990) The "challenge hypothesis": theoretical implications for patterns of testosterone secretion, mating systems, and breeding strategies. Am Nat 136:829-846

Yasmin SE, Yahya HSA (1996) Correlates of mating success in Indian peafowl. Auk 113:490-492

Zahavi A (1975) Mate selection-a selection for a handicap. J Theor Biol 53:205-213 